07,13

\title{
Статистический учет влияния шероховатости на значения твердости, измеряемые при помощи метода инструментального индентирования
}

\author{
(С) И.И. Маслеников ${ }^{1}$, А.С. Усеинов ${ }^{1}$, К.С. Кравчук ${ }^{1}$, А.А. Косцова ${ }^{1}$, В.Н. Решетов ${ }^{2}$ \\ ${ }^{1}$ Технологический институт сверхтвердых и новых углеродных материалов, \\ Москва, Троицк, Россия \\ ${ }^{2}$ Московский инженерно-фризический институт, \\ Москва, Россия \\ E-mail: i.i.maslenikov@gmail.com
}

\begin{abstract}
Рассмотрена возможность корректировки значений твердости шероховатой поверхности, измеряемых при помощи метода инструментального индентирования. Коррекция значений предполагает использование данных о рельефе поверхности, полученных при помощи атомно-силового микроскопа или иного инструмента. Поверхность описывается при помощи стандартного отклонения распределения высот, а также автокорреляционной функции, которую предлагается аппроксимировать экспоненциальной зависимостью с квадратичным или линейным показателем.
\end{abstract}

Работа выполнена при финансовой поддержке Министерства образования и науки Российской Федерации в рамках соглашения № 14.577.21.0274 (уникальный идентификатор проекта RFMEFI57717X0274).

DOI: $10.21883 /$ FTT.2018.11.46666.15NN

\section{1. Введение}

Метод инструментального индентирования [1] широко распространен для измерения механических свойств материалов в субмикронных масштабах при исследовании гетерогенных структур и тонких покрытий. В противоположность традиционному методу определения твердости по оптическому изображению остаточного отпечатка, для получения значения контактной площади в методе инструментального индентирования измеряется зависимость приложенной нагрузки от заглубления индентора при вдавливании в поверхность. При этом используемая для обработки данных модель предполагает, что поверхность является гладкой и ровной. Данное предположение реализуется на практике через требование к проводимым измерениям, при котором глубина внедрения индентора не может быть менее $20 \cdot R a$, где $R a$ - это среднеарифметическое отклонение точек поверхности от средней линии (плоскости). Вместе с тем, при необходимости измерений с небольшими глубинами индентирования подготовка поверхности становится все более затруднительной, а наличие шероховатости приводит не только к увеличению разброса данных, но также и к смещению оценки среднего значения измеряемых величин.

Основной величиной, подверженной влиянию шероховатости, является расчетное значение проекции контактной площади $A(h)$, являющееся функцией контактной глубины $h$. Возможные пути коррекции данной неточности предполагают коррекцию глубины $h$ [2,3] $h \rightarrow h-\langle d z\rangle$, либо коррекцию самой функциональной зависимости $A(h)$ [3]: $A(h) \rightarrow m \cdot A(h)$. Данные поправки позволяют вычислять значение твердости используя стандартную процедуру метода инструментального индентирования с приведенными выше заменами. Указанные способы коррекции соответствуют двум предельным случаям. В первом предельном случае предполагается, что углубления достаточно велики: настолько, что в область контакта попадает достаточно много локальных пиков, которые были смяты гранями индентора, что фактически сводит ситуацию к взаимодействию с плоской поверхностью, смещенной относительно начальной точки касания на величину $\langle d z\rangle$. Во втором предельном случае рассматривается взаимодействие с частью одного локального пика шероховатости, т. е. предполагается, что поверхность является локально плоской, но наклонённой, что и приводит к замене $A \rightarrow m \cdot A$.

В работе [3] указанные выше коэффициенты были определены в предположении, что автокорреляционная функция имеет вид: $C_{d r}^{(2)} \sim \exp \left(-d r^{2} /\left(2 \sigma_{x y}^{2}\right)\right)$. Вместе с тем, наряду с предположением о гауссовых формах автокорреляционной функции и спектральной плотности мощности шума, интерес представляет также автокорреляционная функция с линейным показателем вида $C_{d r}^{(1)} \sim \exp \left(-d r /\left(2 \sqrt{ } 2 \sigma_{x y}\right)\right)$, для которой спектральная плотность мощности шума имеет вид $\sim 1 /\left(1+(4 \sqrt{ } 2 \pi f)^{2}\right)$. Как следует из экспериментальных данных, данный вид автокорреляционной функции лучше соответствует поверхностям, полученным при обработке методами шлифовки с использованием абразива (имеющим протяженные линейные дефекты в виде борозд от шлифпорошка или наждачной бумаги). Наряду с рассмотрением данной автокорреляционной функции в рамках настоящей работы также проведена экспериментальная проверка возможности коррекции $A \rightarrow m \cdot A$.

\section{2. Определение новой корректировочной функции}

Аналогично подходу работы [3] на основе представленной выше зависимости $C_{d r}^{(1)}$ было сгенерировано 7500 
$a$

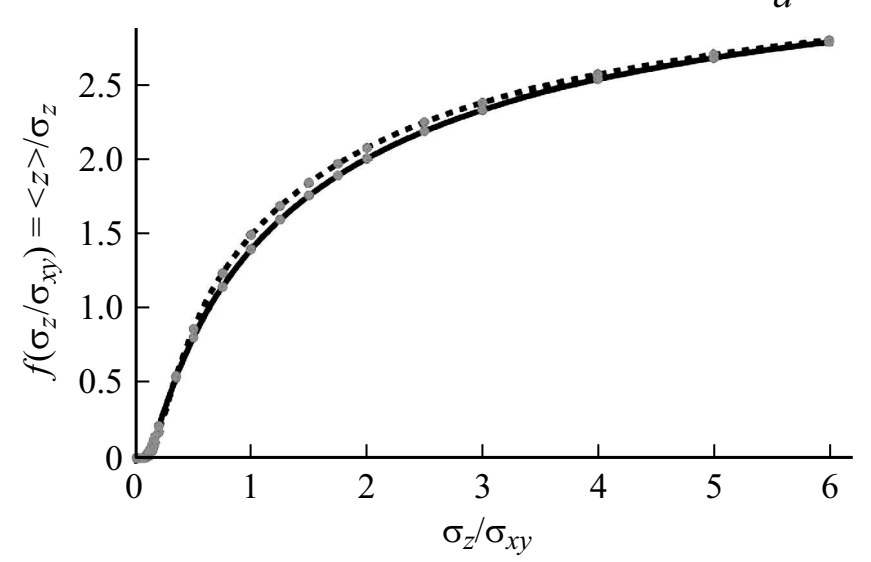

$b$

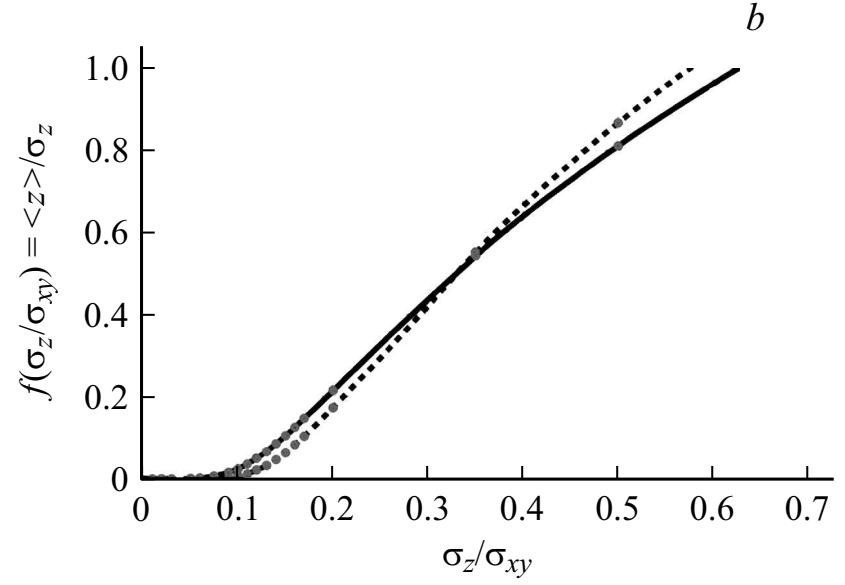

Рис. 1. Зависимость смещения точки контакта от параметра $\sigma_{z} / \sigma_{x y}: a-$ в диапазоне значений $\sigma_{z} / \sigma_{x y}$ от 0 до $6 ; b-$ увеличенная начальная область для диапазона $\sigma_{z} / \sigma_{x y}$ от 0 до 0.7. Пунктирная линия - известная ранее зависимость для функции $C_{d r}^{(2)}$. Сплошная линия - зависимость для функции $C_{d r}^{(1)}$.

шероховатых поверхностей, каждая из которых обладала нормальным распределением высот поверхности со среднеквадратическим отклонением $\sigma_{z}=1$. В данном случае моделирование поверхностей было проведено в безразмерных единицах, так как рассматривается чисто геометрическая задача. Как следует из работы [3], распределение такой величины как, например, смещение точки контакта, является достаточно близким к нормальному, что указывает на то, что погрешность величин, полученных из численного эксперимента, масштабируется как $\sim 1 / \sqrt{ }(N)$, где $N$ - число проведенных измерений. Это позволяет уменьшить итоговую погрешность почти в 100 раз. Учитывая, что целью данной работы было определение именно нормированных параметров, отмеченный факт приводит к тому, что полученные значения будут иметь ошибку не более нескольких процентов: в частности, нормированное на стандартное отклонение смещение точки контакта с вероятностью порядка 95\% будет иметь погрешность не более $2 \%$. Конкретное значение 7500 было выбрано для соответствия условий численного эксперимента данной работы. Каждый из сгенерированных массивов содержал $128 \times 128$ равномерно расположенных точек, параметр $\sigma_{x y}$ был равен четырем единичным расстояниям между соседними точками. Таким образом, исходное соотношение $\sigma_{z} / \sigma_{x y}$ составляло 0.25 для каждого из 7500 массивов. Затем вертикальная координата точек каждого массива была помножена на постоянную для заданного массива константу, изменяя параметр $\sigma_{z}$ таким образом, чтобы получить 29 различных соотношений $\sigma_{z} / \sigma_{x y}$ в диапазоне от 0.01 до 6. Над центром каждой из представленных поверхностей помещалась пирамида в форме Берковича, которая подводилась до первой точки контакта с поверхностью. По полученным расстояниям от средней плоскости шероховатой поверхности до точки контакта было определено среднее значение $\langle d z\rangle$, являющееся функцией параметра $\sigma_{z} / \sigma_{x y}$. Нормированное значение $\langle d z\rangle$, полученное для автокорреляционной функции $C_{d r}^{(1)}$, а также известная ранее зависимость смещения точки контакта для функции $C_{d r}^{(2)}$ представлены на рис. 1. Интересным фактом является практическое совпадение функций $f^{(2)}(x)$ и $f^{(1)}(x)$ : их различие наблюдается только в области малых отношений $\sigma_{z} / \sigma_{x y}$ и не превышает величины порядка $10 \%$ в области $\sigma_{z} / \sigma_{x y}>0.2$. Данная величина примерно соответствует котангенсу угла между ребром и высотой пирамиды Берковича. При больших значениях параметра $\sigma_{z} / \sigma_{x y}$ автокорреляционные функции $C_{d r}^{(2)} \sim \exp \left(-d r^{2} /\left(2 \sigma_{x y}^{2}\right)\right)$ и $C_{d r}^{(1)} \sim \exp \left(-d r /\left(2 \sqrt{ } 2 \sigma_{x y}\right)\right)$, вид которых приведен на рис. 2 , определяют практически одинаковую коррекцию точки контакта.

Представленный теоретический результат позволяет подбирать правильную коррекцию точки контакта для

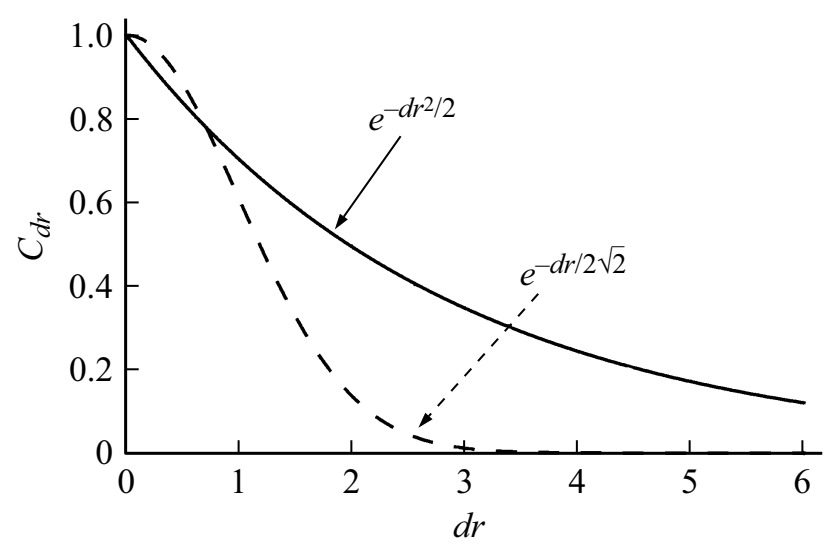

Рис. 2. Сечения автокорреляционных функций, приводящих к одинаковой коррекции точки контакта при больших значениях параметра $\sigma_{z} / \sigma_{x y}$. 
автокорреляционной функции, имеющей отличный от гауссового вид.

\section{3. Экспериментальное исследование}

Представленная модель, выраженная зависимостью $f^{(1)}(x)$, была использована для коррекции экспериментальных данных, измеренных на образцах поликарбоната согласно методу инструментального индентирования. Подготовка поверхности проводилась при помощи наждачной бумаги с различной степенью зернистости, параметр которой приведен в табл. 1. Указанные параметры зернистости были подобраны с целью получения поверхностей с достаточно большим отношением $\sigma_{z} / \sigma_{x y}$ : при $\sigma_{z} / \sigma_{x y} \geq 0.1-$ в области, где поправки, связанные со смещением точки контакта, не пренебрежимо малы (см. рис. 1). При этом твердость поликарбоната позволяет производить измерения с доступными в нанотвердомере „НаноСкан 4Д““ [4] нагрузками на достаточную по сравнению с шероховатостью глубину. Наблюдения при помощи оптического микроскопа не выявило следов шаржирования поверхности. Измерение топографии поверхности было проведено при помощи атомно-силового микроскопа (ACM) „NTegra Prima“, поле сканирования составило более $240 \mu \mathrm{m}$. Параметр $\sigma_{z}$ был определен как среднеквадратическое отклонение всех вершин профиля поверхности. Параметр $\sigma_{x y}$ был определен на основе вписывания теоретических зависимостей в экспериментальные автокорреляционные функции (рис. 3).

Для оценки возможности описания автокорреляционных функций зависимостью $C_{d r}^{(2)}$ на рис. 4 приведена поверхность, полученная аппроксимацией экспериментальных пиков рис. 3 соответствующей гауссовой кривой $C_{d r}^{(2)}$.

Из сравнения аппроксимаций рис. 3 и 4 можно отметить, что экспериментальный пик является более „острым“, чем гауссовы зависимости $\operatorname{Cdr}(2)$. Количественной мерой остроты пика может служить коэффициент эксцесса, определенной зависимостью $\kappa=\mu_{4} / \mu_{2}^{2}-3$, где $\mu_{4}$ - центральный момент четвертого порядка, $\mu_{2}-$ центральный момент второго порядка. Для нормального распределения $C_{d r}^{(2)}$ имеет место равенство $\kappa=0$, для распределения $C_{d r}^{(1)}: \kappa=3$. При этом в обоих случаях нормировочная константа $c_{0}$ была выбрана таким образом, чтобы выполнялось равенство: $\int_{0}^{\infty} c_{0} C_{d r}^{i} d r=1$, где $i=1$, 2. Значения параметра $\kappa$ для экспериментальных массивов, представленных на рис. 3 и 4, приведены в табл. 1. При вычислениях данного параметра в каждом случае использовалась центральная область экспериментальной автокорреляционной функции с радиусом $10 \cdot \sigma_{x y}$, где параметр $\sigma_{x y}$ определен на основании аппроксимаций $C_{d r}^{(1)}$, представленных на рис. 3 .

Таким образом, автокорреляционные функции для всех поверхностей оказались более „острыми“, чем
Таблица 1. Значения параметра эксцесса для экспериментальных автокорреляционных функций

\begin{tabular}{c|l}
\hline $\begin{array}{c}\text { Параметр } \\
\text { зернистости }\end{array}$ & \multicolumn{1}{c}{$\kappa$} \\
\hline 180 & 0.57 \\
320 & 3.34 \\
800 & 7.3
\end{tabular}

Таблица 2. Результаты измерения твердости поверхности поликарбоната с различной шероховатостью

\begin{tabular}{c|c|c|c|c}
\hline $\begin{array}{c}\text { Параметр } \\
\text { зернистости }\end{array}$ & $\sigma_{z} / \sigma_{x y}$ & $F, \mathrm{mN}$ & $\begin{array}{c}H(\langle h\rangle) \text { без } \\
\text { коррекции, GPa }\end{array}$ & $\begin{array}{c}H(\langle h\rangle) \mathrm{c} \\
\text { коррекцией, GPa }\end{array}$ \\
\hline \multirow{2}{*}{180} & 0.51 & 100 & 0.08 & 0.2 \\
\hline \multirow{2}{*}{320} & 0.27 & 1400 & 0.15 & 0.2 \\
\hline \multirow{2}{*}{800} & \multirow{2}{*}{0.55} & 1400 & 0.12 & 0.16 \\
& & 1400 & 0.17 & 0.19 \\
\hline
\end{tabular}

гауссова зависимость $C_{d r}^{(2)}$. С увеличением параметра зернистости наждачной бумаги описание поверхности при помощи функции $C_{d r}^{(2)}$ становится все менее адекватным, в то время как зависимость $C_{d r}^{(1)}$ позволяет более корректно описать острый пик.

Для дополнительного качественного объяснения можно отметить, что в окрестности точки $d r=0$ зависимость $C_{d r}^{(1)}$ в отличие от зависимости $C_{d r}^{(2)}$ является линейной относительно параметра $d r$; аналогичное линейное разложение будет иметь место и для пиков автокорреляционных функций прямоугольных или треугольных сигналов. Данные сигналы являются качественным приближением сечений борозд поверхности, получаемых при фрезеровке или шлифовке, что и приводит к качественному выводу о том, что автокорреляционные функции таких поверхностей лучше описываются именно зависимостью $C_{d r}^{(1)}$ с линейным показателем в экспоненте.

Для измерения твердости использовался нанотвердомер „НаноСкан 4Д“ [4], на каждом из образцов было проведено 100 измерений. Контактные глубины для каждой из нагрузок были усреднены; итоговое значение твердости без коррекции было рассчитано как $F / A(\langle h\rangle)$, с учетом коррекции твердость рассчитывалась как $F / A(\langle h\rangle-\langle d z\rangle)$. Параметр $\sigma_{z}$ для указанных поверхностей составил от $1.1 \mu \mathrm{m}$ до $3.3 \mu \mathrm{m}$. Результаты измерений представлены в табл. 2.

Как видно из таблицы, представленная модель во всех случаях существенно улучшает точность определения значения твердости, приближая его к твердости ровной поверхности $(0.2 \mathrm{GPa})$. Так как методика предполагает 

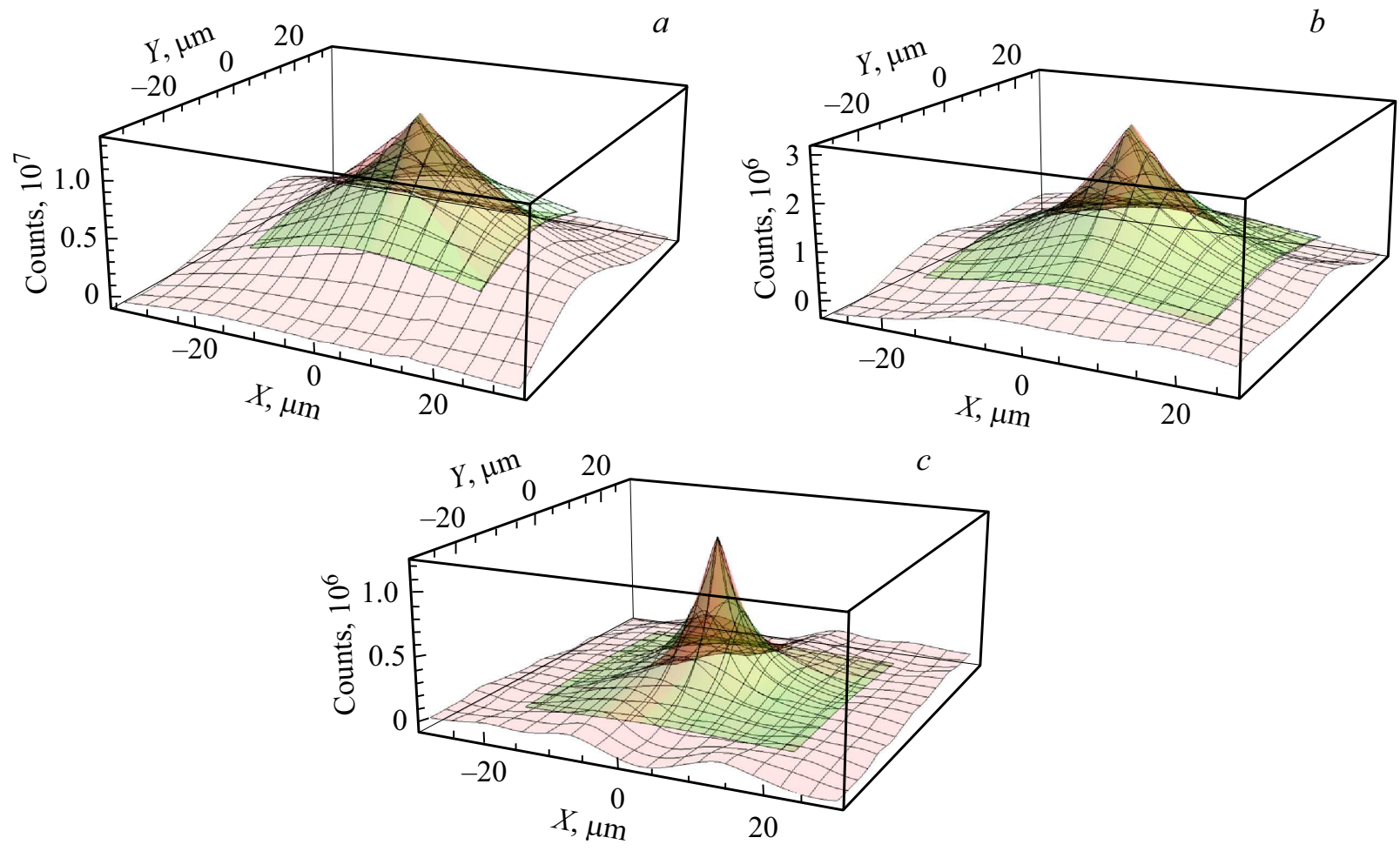

Рис. 3. Автокорреляционные функции, а также аппроксимирующие их зависимости $C_{\mathrm{dr}}^{(1)}$, полученные для поверхностей, соответствующих параметру зернистости: $180(a), 320(b), 800(c)$.
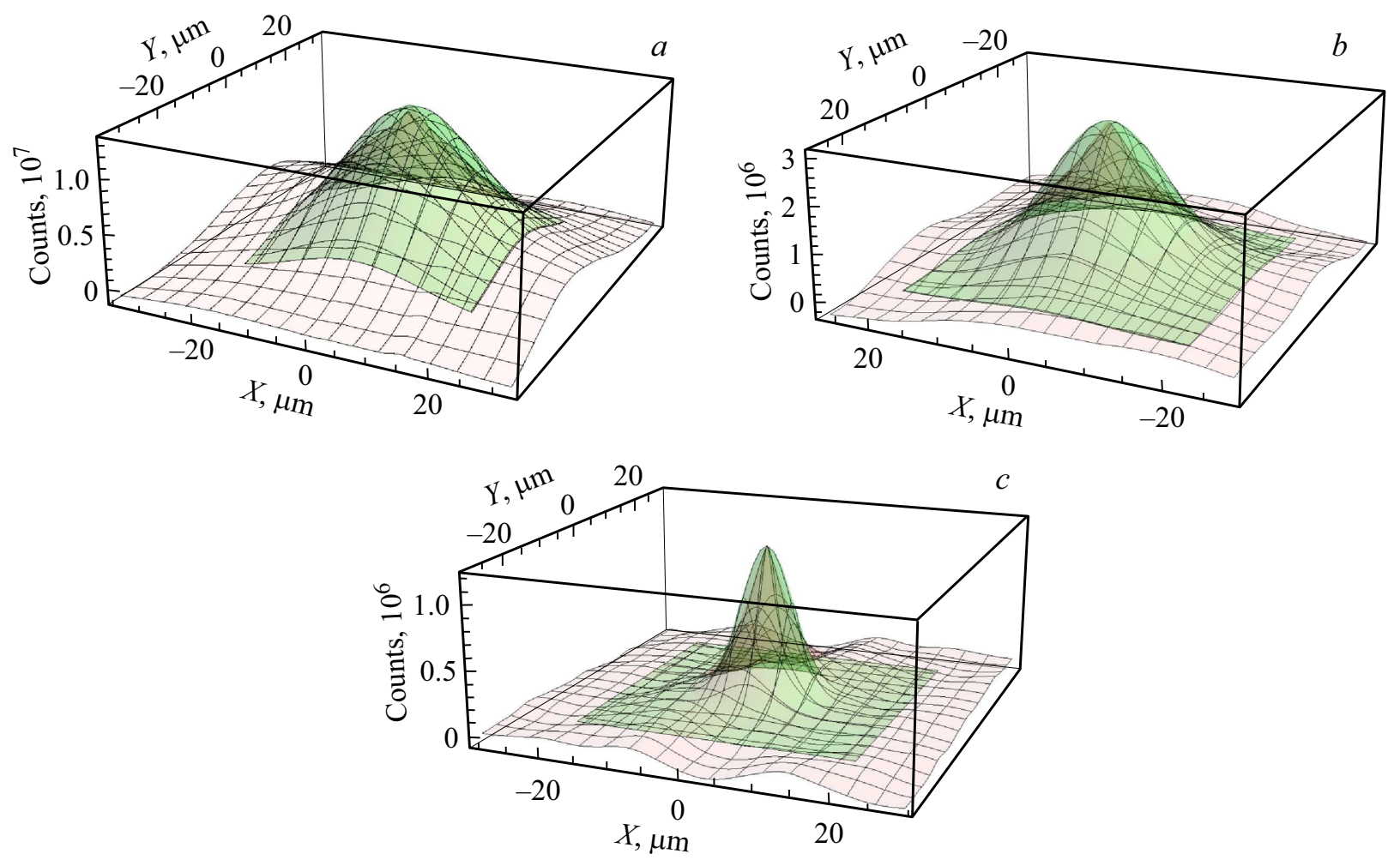

Рис. 4. Автокорреляционные функции, а также аппроксимирующие их зависимости $C_{\mathrm{dr}}^{(2)}$, полученные для поверхностей, соответствующих параметру зернистости: $180(a), 320(b), 800(c)$. 
$a$
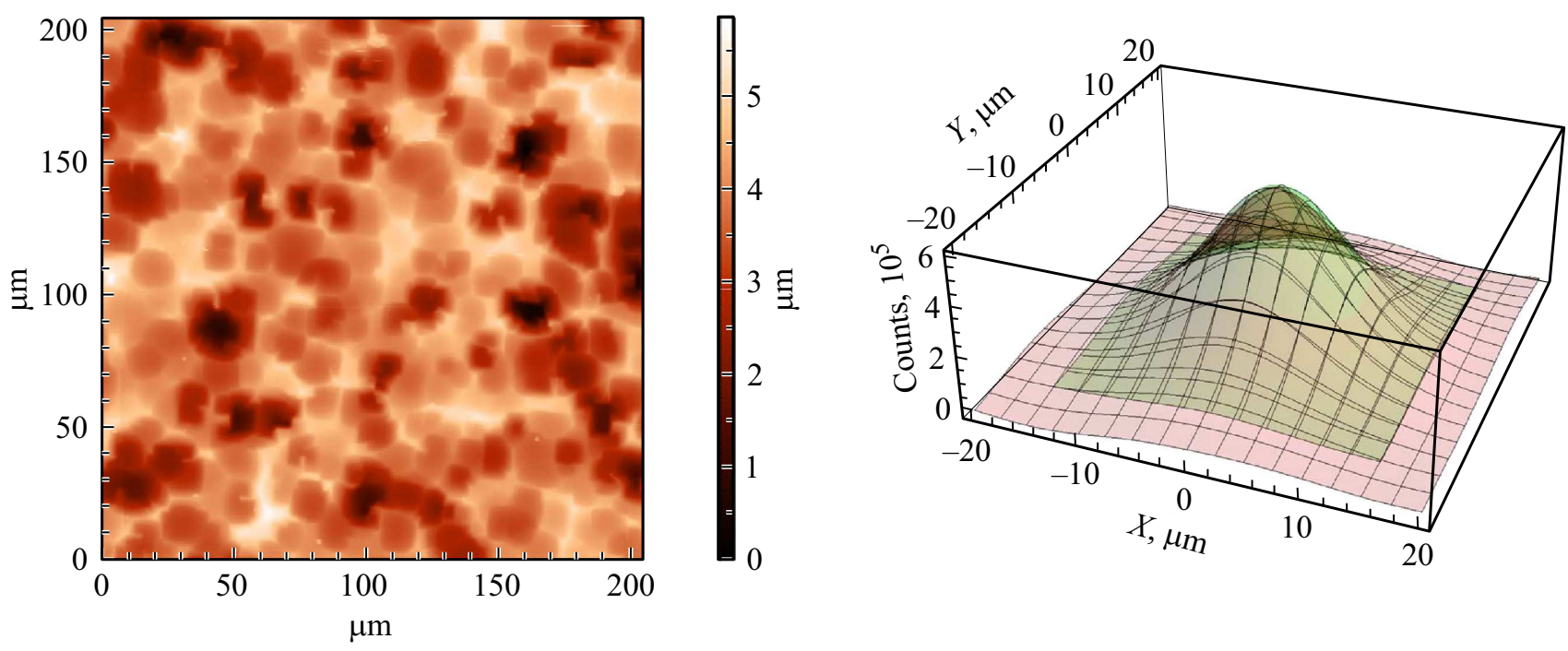

Рис. 5. АСМ-изображение поверхности кремния $(a)$, автокорреляционная функция и аппроксимирующая ее поверхность $(b)$.

коррекцию среднего значения глубины, а затем вычисление твердости, то сравнение производилось именно с твердостью, вычисленной от средней глубины без коррекции. Следует отметить, что усреднение значений твердости, вычисленных от каждого значения контактной глубины, также приводит к значению, большему значения твердости, вычисленному от средней глубины, однако такой способ не гарантирует получения значения твердости, соответствующего ровной поверхности.

В дополнение к коррекции твердости, произведенной для относительно больших значений $\sigma_{z} / \sigma_{x y}>0.2$, была исследована возможность экспериментальной коррекции $A \rightarrow m \cdot A$, позволяющей корректировать значения твердости вПлоть до значений $\sigma_{z} / \sigma_{x y} \sim 0.1$. В качестве образца была выбрана пластина кремния, изображение и автокорреляционная функция поверхности которой приведены на рис. 5. Экспериментальная автокорреляционная функция хорошо описывается гауссовой кривой $C_{d r}^{(2)} \sim \exp \left(-d r^{2} /\left(2 \sigma_{x y}^{2}\right)\right)$ с параметром $\sigma_{x y}=6.5 \mu \mathrm{m}$. Среднеквадратичное отклонение рельефа поверхности $\sigma_{z}$ составило $0.76 \mu \mathrm{m}$. Индентирование было произведено с нагрузкой $15 \mathrm{mN}$, что привело к значению средней контактной глубины $0.22 \mu \mathrm{m}$. Применение коррекции $A(\langle h\rangle) \rightarrow m \cdot A(\langle h\rangle)$ с приведенным в работе [3] значением параметра $m=1+14.2\left(\sigma_{z} / \sigma_{x y}\right)^{2}$ позволило скорректировать исходное значение твердости $F / A(\langle h\rangle)=9.1 \mathrm{GPa}$ до значения $10.8 \mathrm{GPa}$; измеренное значение твердости ровной поверхности составило $11.3 \pm 0.5 \mathrm{GPa}$. Таким образом показано, что в результате применения предложенной коррекции значение твердости шероховатой поверхности совпало со значением для ровной поверхности в пределах погрешности измерения.

\section{4. Заключение}

В рамках данной работы была выведена зависимость, позволяющая корректировать значения твердости при больших глубинах индентирования в случае, когда автокорреляционная функция имеет вид $C_{d r}^{(1)}$. В отличие от использованной ранее зависимости $C_{d r}^{(2)}$, указанный вид автокорреляции более соответствует поверхностям, содержащим выраженный рельеф, характерный для обработки методами шлифовки или фрезеровки. Полученная зависимость была проверена экспериментально. Также экспериментальная проверка была проведена и для индентирования на малые глубины в случае поверхности, имеющей гауссову автокорреляцию высот. Полученные данные демонстрируют возможность коррекции измеряемых значений твердости с целью повышения точности определения механических свойств с помощью метода инструментального индентирования.

\section{Список литературы}

[1] W.C. Oliver, G.M. Pharr. J. Mater. Res. 19, 1, 3 (2004).

[2] J.Y. Kim, J.J. Lee, Y.H. Lee, J. Jang, D. Kwon. J. Mater. Res. 21, 12, 2975 (2006).

[3] I. Maslenikov, A. Useinov, A. Birykov, V. Reshetov. IOP Conf. Series: Materials Science and Engineering 256, 012003 (2017).

[4] К. Кравчук, А. Усеинов, И. Маслеников, С. Перфилов. Наноиндустрия 65, 3.54 (2016).

Редактор Т.Н. Василевская 